\title{
ON THE NATURE OF BACTERIAL LAG.
}

By WILLIAM JAS. PENFOLD.

(From the Bacteriological Department, Lister Institute, London.)

\author{
(With 5 Charts.)
}

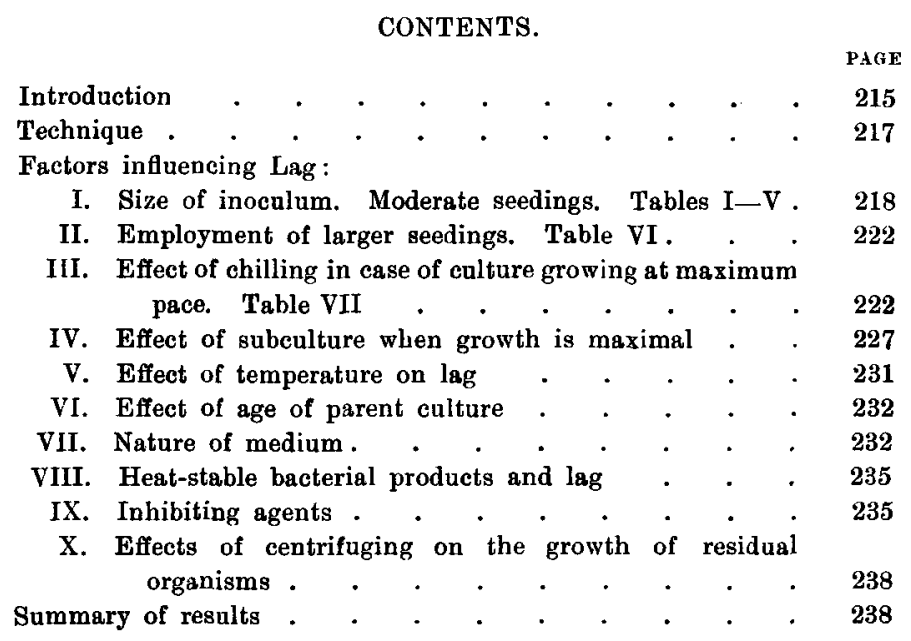

\section{Introduction.}

THE rate of bacterial growth was first measured with any degree of accuracy in the case of the Vibrio cholerae asiaticae growing in broth, by Buchner, Longard and Riedlin (1887). They gave a generation time in the case of this organism of 19 to 40 minutes. These times were calculated from the numbers of those inoculated and of those found to be present in cultures after two to five hours' growth. 
The large differences obtained in different experiments were explained by variations of the strain in artificial culture. Max Müller (1895) drew attention to the fact that the wide variations in the figures of Buchner, Longard and Riedlin were due, not as these authors believed to variations of the strain, but to the fact that the periods of observations varied in length; he pointed out that those cultures which gave long generation times had been allowed to grow only for short periods, while those which gave short generation times had been allowed to grow for longer periods. Further, this author, by a series of counts, was able to demonstrate experimentally the existence of lag.

By bacterial lag, we understand, the interval between the inoculation of a bacterial culture and the time of commencement of its maximum rate of growth. This has also been referred to as latency, restraint of growth, and various other terms.

\section{The measurement of lag.}

(1) The lag may be expressed in terms of the period (hours) during which submaximal growth continues.

(2) Myer Coplans (1909) has expressed it as restraint of growth in terms of minimum generation times. If, for example, $x$ hours were required after inoculation before a culture showed its minimum generation time, and if it then had a generation time of $y$ hours, and the number of generations that actually arose during $x$ was $z$ then $\frac{x-z y}{y}$ $=$ the measure of restraint of growth in terms of minimum generation times.

(3) An index of lag is readily obtained by comparing the average generation time during the first hours of growth with the average generation time of a succeeding period or several such periods.

All these methods have difficulties. The error of measurement of numbers of bacteria present is not inconsiderable, and this circumstance makes the precise definition of the limits of lag difficult.

Where attempts are made to estimate generation time on an increase of bacterial population amounting to $0 \cdot 3$ of a generation the results are very unreliable. On the other hand, where two generations or more have developed during the period under consideration, results of great precision can be obtained The investigation of lag involves a large number of experiments of a comparative character. To diminish the number of variables it is advisable to use the same sample of peptone 
in preparing the peptone-water medium. The temperature must be carefully noted several times during the continuance of each experiment. The organism of course is a factor that we cannot yet keep constant, for which reason it is desirable when endeavouring to ascertain the influence of any one factor on lag that all the comparative experiments be done simultaneously.

Lag is a subject of considerable interest. It occurs in many biological reactions, for example, haemolysis, bacteriolysis and many others, and it seems not impossible that light thrown on any one of these may illumine the rest. Further, the incubation period of infectious disease may partly depend for its existence on bacterial lag.

Satisfactory quantitative work on this subject is small in amount. I intend therefore to submit records of experiments showing in a quantitative manner the influence of various factors on lag.

\section{Technique.}

The culture medium used was always $1.0 \%$ peptone (Witte) $+0.5 \%$ salt. It was sterilized by autoclaving, and its reaction was faintly alkaline to neutral litmus paper. B. coli was the organism employed. It was subcultured every day from peptone water to peptone water of the composition indicated and used generally as a 17 to 20 hours' culture for the inoculation of the peptone water of the actual experiment. The actual experiments were always carried out at $37^{\circ} \mathrm{C}$. unless otherwise stated. The parent cultures were likewise always grown at $37^{\circ} \mathrm{C}$. The standard drop of the experiments was 0.02 c.c.

The agar plating method was used to ascertain the numbers of bacteria in the growing cultures. A small amount of agar was first poured into each plate. When this had set, the quantity of bacterial emulsion was added to the plate and then a whole tube of agar added and mixed with the emulsion. After about five minutes, a further small quantity of agar was added, sufficient to cover the second layer. This method greatly facilitated the counting process as all colonies were discrete, and no spreading occurred. All dilutions of parent cultures and subcultures were made in normal saline solution.

In the following experiments generation time signifies the average generation time during the interval dealt with. If, during the interval, the generation time has been varying, this method of expression is not entirely satisfactory.

In such cases generation time at particular moments is the only absolutely satisfactory expression. The former method has however 
been largely and legitimately used in recorded work. Comparatively few of the recorded experiments on this subject enable one to obtain the generation time, with any accuracy, at particular moments during periods of varying rate of growth, and this precision is not usually necessary. (See Ledingham and Penfold, This Journal, p. 242.)

\section{Factors influencing lag.}

Size of inoculum. Size of inoculation was stated by Rahn (1906) to have an influence on lag. He stated that the greater the inoculum the shorter the lag. Since this appears to have an important bearing on the nature of lag, I examined his evidence carefully. Rahn's figures however will not bear careful scrutiny. In Table I I reproduce his numbers, his experimental data and generation times. It will be noticed that in columns III, IV and V the initial count per c.c. increases from III to $\mathrm{V}$ while the volumes are constant. His calculated generation times, given below, suggest that the maximum lag is obtained in III but this is found to depend on an arithmetical error since, on recalculation, tube III in reality shows its minimum generation time in the same time interval as tube IV. I have introduced certain corrections in arithmetic into his table, these corrections being underlined. In the case of tube $\mathrm{V}$ its lag is shorter than III and IV, but it never attains

\section{TABLE I. [T'abelle III (Page 422).]}

Bacillus fluorescens in Traubenzuckerpeptonlösung. Bakterienanzabl pro c.cm.

\begin{tabular}{|c|c|c|c|c|c|c|c|c|c|}
\hline \multirow{3}{*}{\multicolumn{2}{|c|}{ Flüssigkeitsmenge }} & \multicolumn{2}{|c|}{ I } & II & \multicolumn{2}{|c|}{ III } & IV & \multicolumn{2}{|c|}{$\mathbf{V}$} \\
\hline & & \multicolumn{2}{|c|}{$1000 \mathrm{c.cm}$. } & $10 \mathrm{c.cm}$. & \multicolumn{2}{|c|}{100 c.en. } & $100 \mathrm{c.cm}$. & \multicolumn{2}{|c|}{$100 \mathrm{c.cm}}$. \\
\hline & & \multicolumn{2}{|c|}{30,000} & 30,000 & \multicolumn{2}{|c|}{3,000} & 30,000 & \multicolumn{2}{|c|}{$3,000,000$} \\
\hline \multicolumn{2}{|c|}{ Nach 6 Stunden } & \multicolumn{2}{|c|}{50,500} & - & \multicolumn{2}{|r|}{$[200]$} & - & \multicolumn{2}{|c|}{$5,280,000$} \\
\hline ,, 12 & , & \multicolumn{2}{|c|}{290,800} & $7,610,000$ & \multicolumn{2}{|r|}{$[200]$} & $\mathbf{3 3 0 , 0 0 0}$ & \multicolumn{2}{|c|}{$20,000,000$} \\
\hline , 24 & ," & \multicolumn{2}{|c|}{$6,430,000$} & $82,000,000$ & \multicolumn{2}{|c|}{130,000} & $33,000,000$ & \multicolumn{2}{|c|}{$59,000,000$} \\
\hline , 36 & ," & \multicolumn{2}{|c|}{$10,000,000$} & $180,000,000$ & \multicolumn{2}{|c|}{$13,000,000$} & $70,000,000$ & \multicolumn{2}{|c|}{$82,000,000$} \\
\hline,$\quad 53$ & , & 8,00 &, 000 & $75,000,000$ & 66,0 & 0,000 & $110,000,000$ & 66,00 & 0,000 \\
\hline & & & & Generat & uer. & & & & \\
\hline & & 1 & & II & & II & IV & & $V$ \\
\hline von $0-6 \mathrm{~S}$ & nden & 479 & ains. & 90 mius. & {$[\infty]$} & mins.) & & $462 n$ & mins. \\
\hline , 6-12 & , & 1616 & $, 142\}$ & 90 wins. & {$[\infty]$} &,$y$ & 210 mins. & 187 & $"$ \\
\hline, $12-24$ & ," & 61 & , 161 & 210 & 132 & , 77 & 108 & 461 & ", \\
\hline , $\begin{array}{r}24-36 \\
36-53\end{array}$ & , & 1130 & ", & $\underset{\infty}{634, "}$ & $\begin{array}{l}108 \\
435\end{array}$ & ," & $\begin{array}{r}664 \\
1564\end{array}$ & $\begin{array}{c}1629 \\
\infty\end{array}$ & ", \\
\hline,, $36-53$ & ,", & $\infty$ & & $\infty$ & 435 & $"$ & 1564 & $\infty$ & \\
\hline
\end{tabular}

The underlined numbers are corrections of arithmetic which $I$ have introduced. 
very rapid growth and its short lag is probably really due to the fact that the factors inhibiting growth came into operation before the lag had ceased. The series therefore on which Rahn relied will not bear examination.

It is extremely interesting to note in the same table that I, II and IV form a comparable series to which Rahn did not draw attention. They all have the same initial number per c.c. but, owing to the large difference in volume, the size of inoculum is enormously different. The time figures given after again revising the arithmetic, show that the smallest inoculum has the shortest lag, an opposite conclusion to that drawn by Rahn. A further serious drawback to Rahn's work is the infrequency of observations. It is quite obvious that to establish the minimum generation time of a culture satisfactorily, two, or better still, three, consecutive periods with approximately the same generation time, within the limits of experimental error, are required, or the several tubes of the same strain growing in the same medium should show the same generation time proving that they have attained comparable rates of growth. Rahn's work on the point does not satisfy either of these conditions. The minimum generation times which he found in the five tubes of the same culture medium inoculated with the same organism at the same time, varied widely, as a reference to Table I will immediately show.

The position required further experimental work. For this purpose I tested the effect of size of inoculum on the lag shown by $B$. coli Escherich when grown on peptone water. Table II gives one complete experiment. The experiment was conducted in duplicate and the number per drop determined on the average of the two plates. The sizes of inoculum were approximately as $1: 10,25: 100$. The tubes were warmed to $37^{\circ} \mathrm{C}$. before inoculation. The volumes were constant and all the tubes were put in and out of the incubator together, they are strictly comparable. Chart 1 shows the logarithms of the various numbers plotted against time and it is seen that the curves are very similar in each case.

In Table III are shown the average generation times for the first two hours as against those for the third hour. It will be observed that during the third hour the generation times in the case of all the tubes are constant within the error of experiment, while in the case of the first two hours, the generation time diminishes slightly as we pass from the smaller to the larger seedings.

In Table IV are given the average generation times of the first two hours averaged from two experiments each conducted in duplicate, 


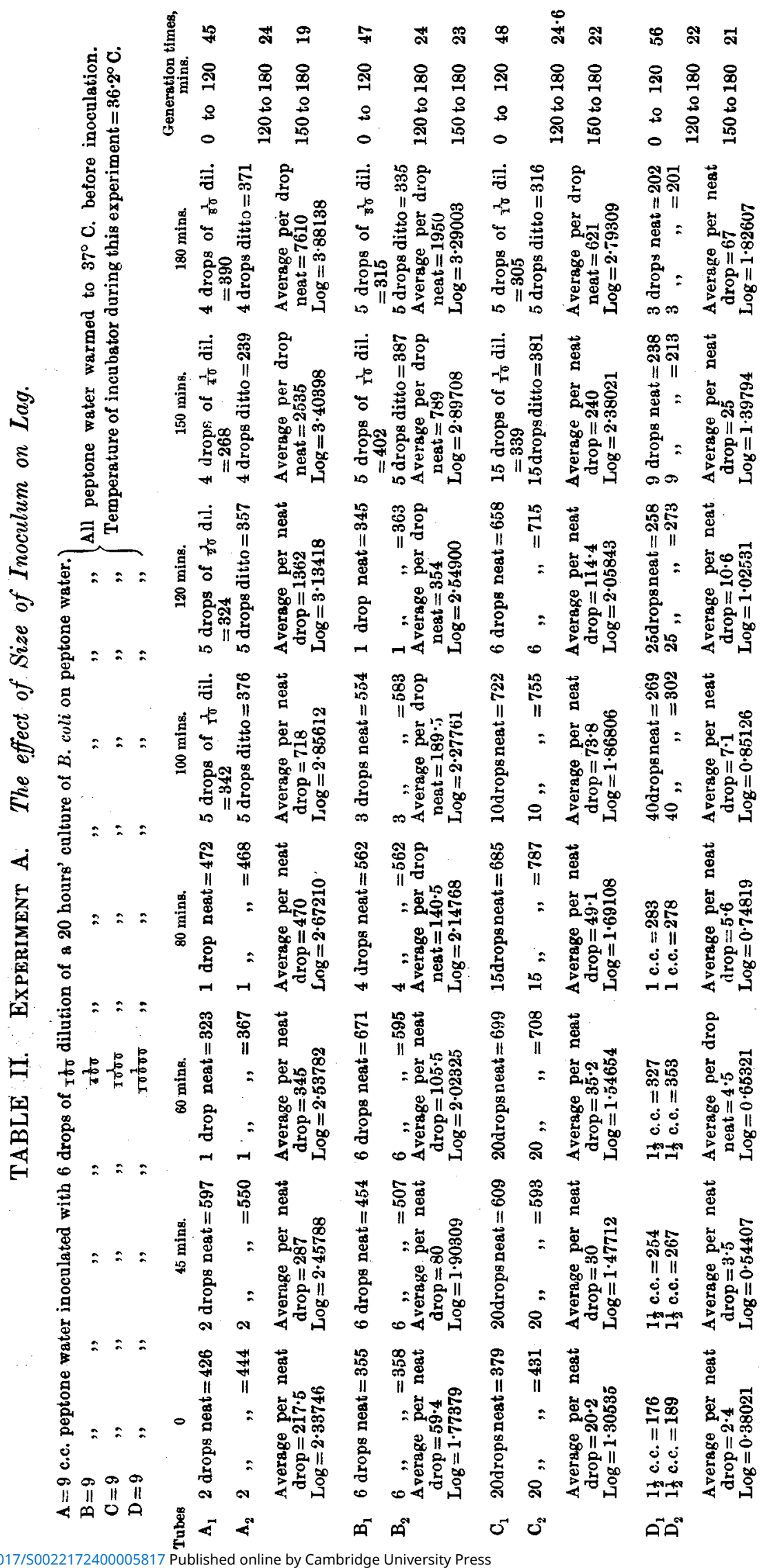


from which it will be seen the larger inoculum definitely tends to grow a little better during the initial period.

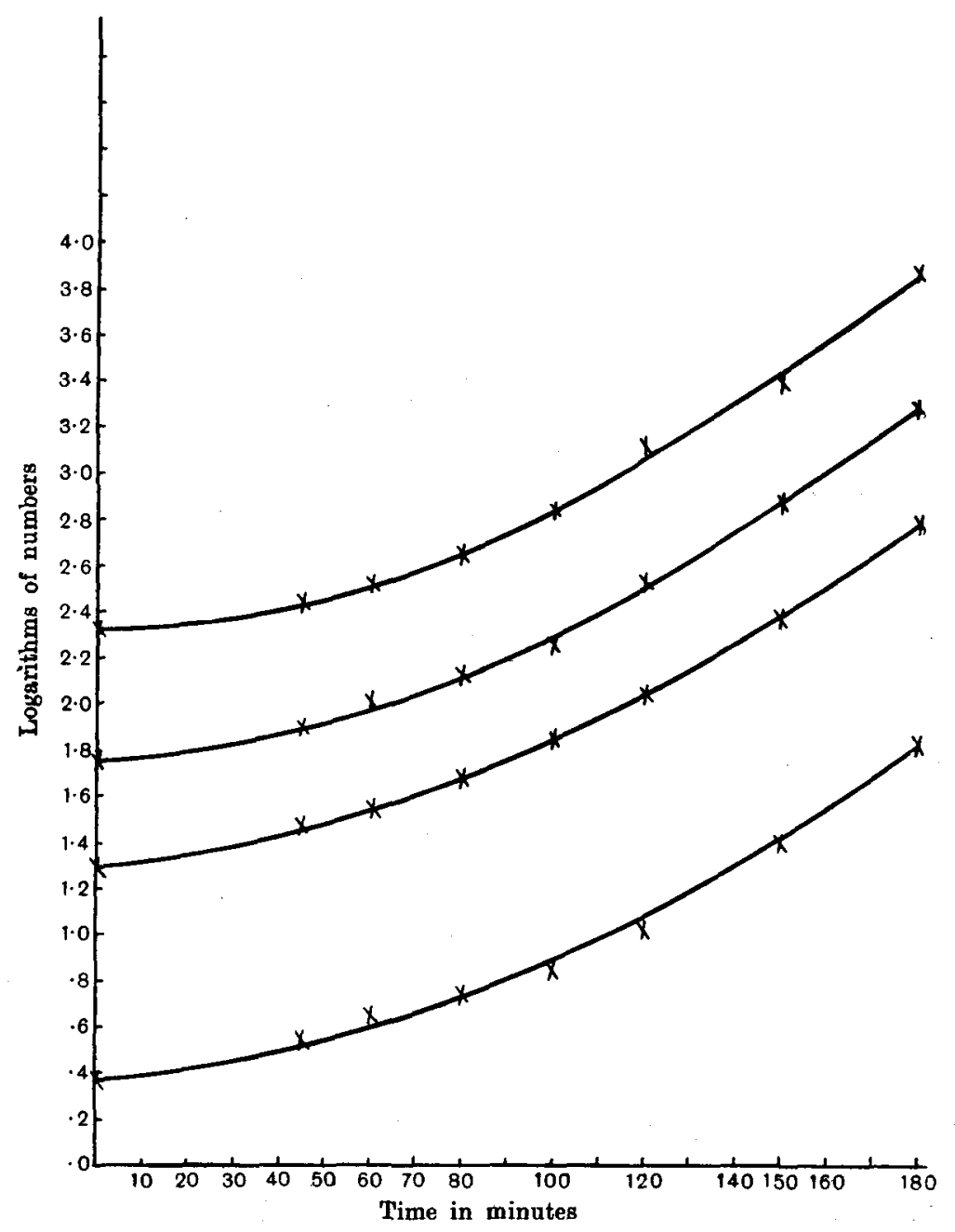

Chart 1. The effect of size of inoculation on lag experiments.

TABLE III.

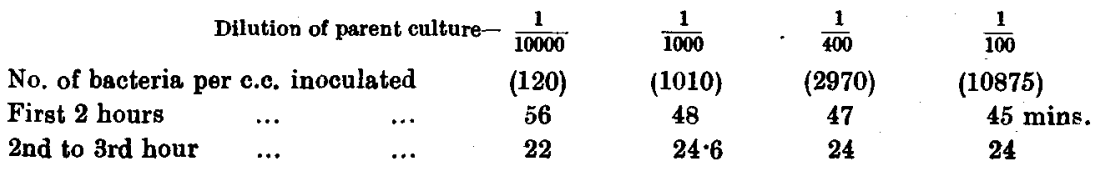


TABLE IV. The generation times of cultures with different inoculums, from two experiments, for the first two hours of growth, with their averages.

\begin{tabular}{cccccc}
\multicolumn{2}{c}{ Dilution of parent culture- } & $\frac{1}{100}$ & $\frac{1}{400}$ & $\frac{1}{1000}$ & 1 \\
Experiment B & $\ldots$ & 44 & 48 & 52 & 91 \\
, , A & $\ldots$ & 45 & 47 & 48 & 56 \\
\hline Average of A and B & $44 \cdot 5$ & $47 \cdot 5$ & 50 & $73 \cdot 5$
\end{tabular}

The details of Experiment B, which was carried out on exactly similar lines to Experiment A, are shown in Table V.

The points in these two experiments were determined on large counts so that the figures might be used for the mathematical treatment of this early portion of the growth curve. (See Ledingham and Penfold, This Journal, p. 242.)

\section{Employment of larger seedings.}

An endeavour was made to elucidate the effect of size of inoculum on lag, in the case of much bigger seedings. The initial population in the case of the largest reached nearly 200,000 per c.c.

One such experiment is recorded in Table VI. This, taken in conjunction with the protocols of the preceding experiments on the same subject, shows that as the inoculum is increased, the diminution of the lag becomes less and ultimately practically disappears. The generation times of the A and B experiments of Table IV for the preliminary two hours of cultures from dilution $\frac{1}{10} \overline{0}$ are practically identical with that obtained in the case of the corresponding culture from ${ }_{1}^{\frac{1}{0}} \overline{0}$ dilution in Experiment C, Table VI, so that these experiments may be reasonably looked upon as one. The experiments were not carried further than a three hours' observation period because, as is seen in Table II, the minimum generation time was attained during the last half hour in each case within the experimental error, viz. 19 to 23 minutes.

Effect on lag of sudden chilling of a culture growing at maximum pace.

The object of these experiments was to see if stoppage by cold, of growth at its maximum, was followed by a lag, when the temperature of the culture was raised again suddenly to its original height. $B$. coli was the organism used and peptone water the medium. Three cultures 





were inoculated from a dilution in saline of a 17 hours' peptone water culture of $B$. coli and all were grown at $37^{\circ} \mathrm{C}$.

(1) The first was kept at $37^{\circ}$ C. throughout as control.

(2) The second culture, after it had grown two hours, was chilled for twelve minutes at $2^{\circ} \mathrm{C}$.; it was then heated suddenly during two minutes in a water bath up to $37^{\circ} \mathrm{C}$. and plated, after which the containing tube was quickly dried and placed in the incubator at $37^{\circ} \mathrm{C}$. Plating of samples followed from time to time. The sudden cooling stopped growth but on being heated again to $37^{\circ} \mathrm{C}$. maximum growth was resumed without lag.
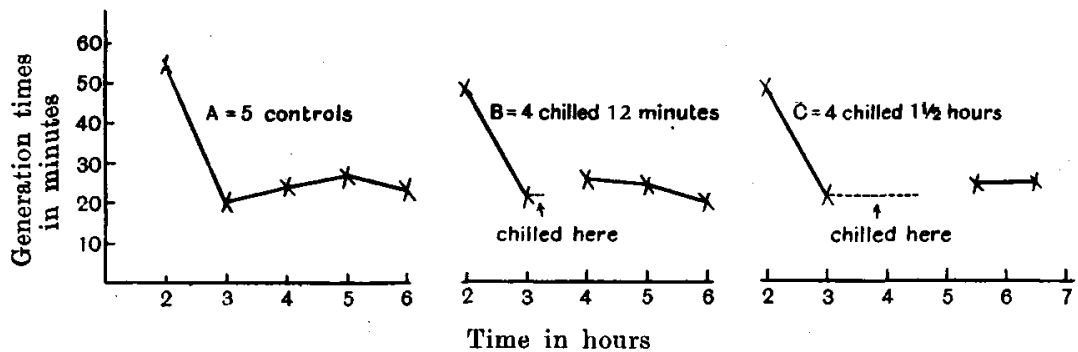

Chart 2a. Chilling during maximum growth and lag.

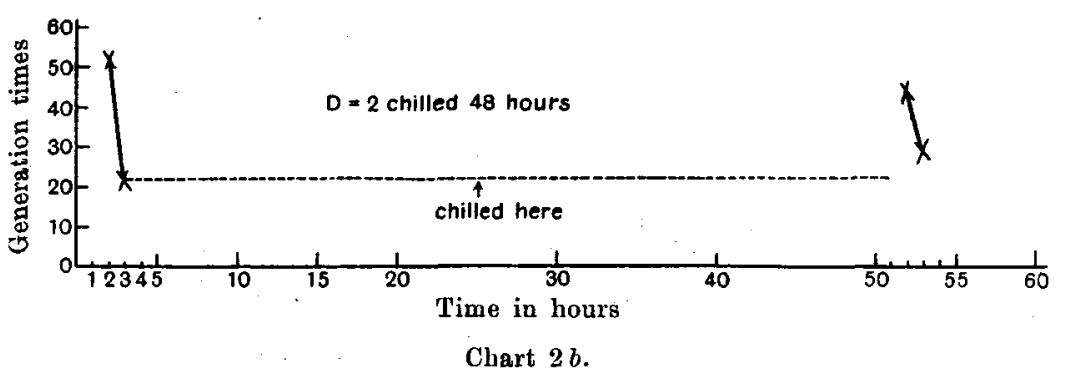

(3) The third culture was cooled for a longer period, viz. one hour and a half. On being heated again to $37^{\circ} \mathrm{C}$., it grew entirely without lag during the first hour. Five such experiments were done. One is recorded in full detail in Table VII. A résumé of four is given in brief in Table VIII and from these figures Chart 2 has been constructed.

The fifth chart was not considered in making the averages as it was an early experiment and did now show all the necessary data.

In two of the experiments Culture III, during the process of chilling, was halved and one half placed in the refrigerator till the 2nd following day. It was then again suddenly heated to $37^{\circ} \mathrm{C}$. and counted by 


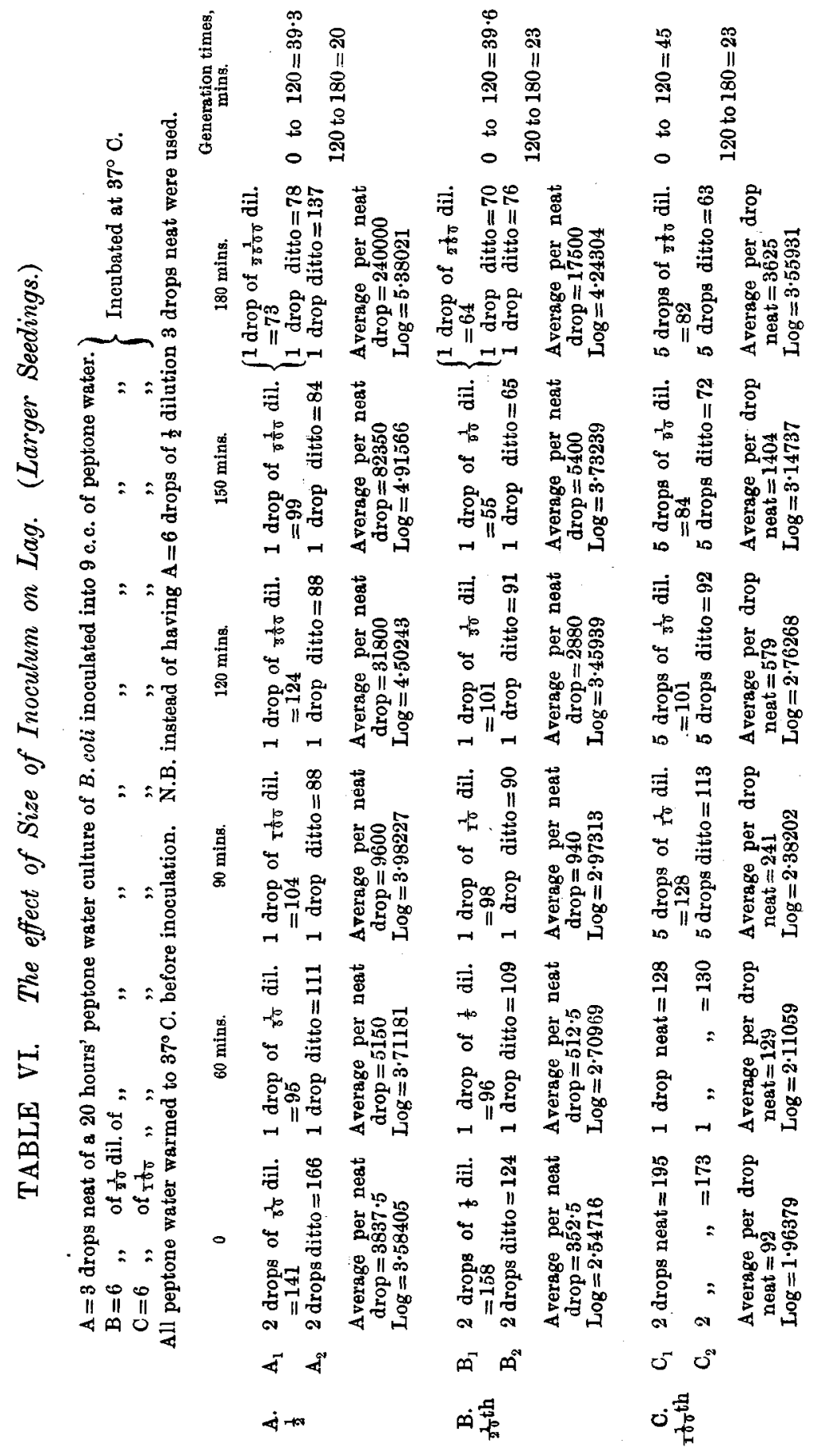

Journ. of Hyg. xiv 


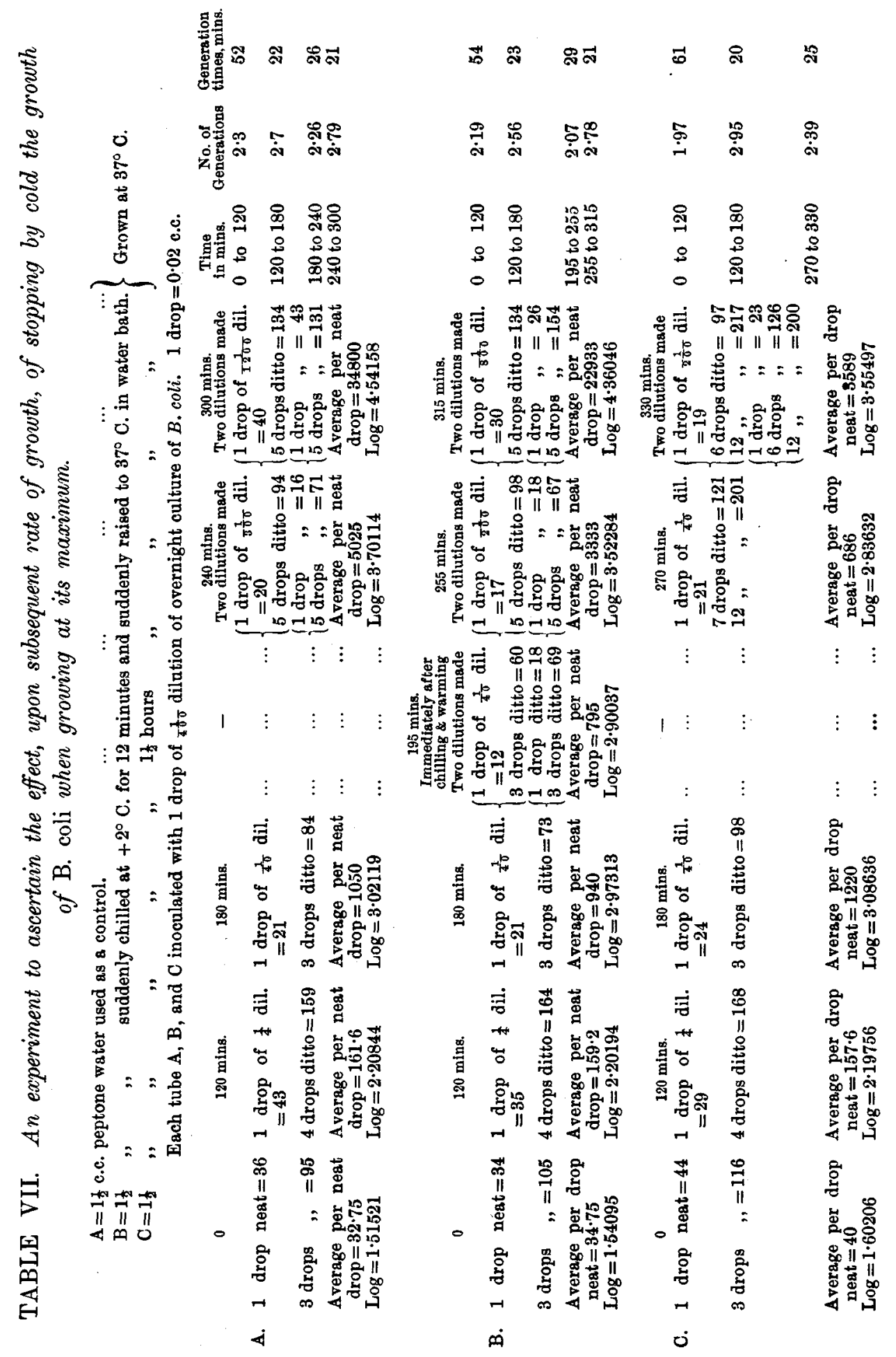


plating. Immediately thereafter it was placed in the incubator, and counted again after one hour and two hours' growth respectively. In this case the numbers are small, especially of the last count, but in the case of the first and second counts, see Table IX and Chart $2 b$, they are sufficient to show that the lag has reappeared, the generation times during the first hour after chilling being 44 minutes while in the case of the evanescent chilling only 21 and 24 minutes were required for one generation during the same interval. We may therefore state that if maximum growth be inhibited by a short application of cold it will recommence immediately without lag on the cold being removed. If, on the other hand, the cold be long continued the lag tends to reappear.

It appeared possible that the mere stoppage of growth in parent cultures might be of itself sufficient to introduce lag, but that possibility is negatived by these experiments. The averages of the experiments on this subject are given in Table VIII.

TABIE VIII. Experiment to show the average generation time during different intervals of peptone water eultures of $\mathrm{B}$. coli Esch., 5 allowed to grow freely at $37^{\circ} \mathrm{C}$. as controls and 10 subjected to chilling.

Average generation time in mins.

\begin{tabular}{lcc||ccc} 
& $\begin{array}{c}\text { During first two } \\
\text { hours of growth }\end{array}$ & $\begin{array}{c}\text { During third } \\
\text { hour of growth }\end{array}$ & 4th hour & 5th hour & 6 th hour \\
5 controls & $55 \cdot 4$ & $20 \cdot 6$ & $24 \cdot 6$ & 27 & $23 \cdot 5$ \\
$\begin{array}{l}4 \text { cultures cooled } \\
12 \text { mins. each }\end{array}$ & $48 \cdot 5$ & $21 \cdot 75$ & $25 \cdot 7$ & 24 & 20 \\
4 cooled 1 1 hours & $48 \cdot 5$ & $21 \cdot 25$ & $24 \cdot 5$ & 25 & \\
2 cooled 2 days & 52 & 22 & 44 & 29
\end{tabular}

The double line indicates the time of application of cold.

Subculture when growth is maximum.

The effect of subculture when growth is occurring at maximum pace has been investigated by several writers.

Rahn (1906) states that lag occurs under these conditions but adduces no satisfactory evidence in support of the assertion.

Myer Coplans (1909) agrees with Rahn's view that lag follows subculture during maximum growth. He bases his opinion on the fact that when he subcultured a parent culture of $B$. coli of $14 \frac{1}{2}$ hours' growth (see Chart $P$ in the author's paper) a lag occurred. He further states that a peptone water culture of $B$. coli of 12 hours' age whether 
grown at $20^{\circ}$ or $37^{\circ} \mathrm{C}$. is still in maximum development. Such a generalization is not permissible since the size of inoculation and age of parent culture and other factors must be considered in each special case.

It will also be seen from consulting Table p.p. 1 of his paper, page 4, that that assertion did not hold in this case for the average generation time between $11 \frac{1}{4}$ and $14 \frac{1}{2}$ hours is nearly twice as long as that between 8 hours and $11 \frac{1}{4}$ while the generation time at the end of this interval would be longer still. Without therefore direct evidence of the rate at which the parent culture grew after removal of the sample this question cannot be satisfactorily decided.

I have made six such experiments, the details of two of which are given on the annexed Table $X$. $B$. coli and peptone water were again used. The total volumes of the cultures were 6 c.c. The temperature of the experiments was $37^{\circ} \mathrm{C}$.

The following points emerge from the consideration of Table $\mathrm{X}$. The parent culture had an average generation time during the first two hours of 47 minutes, from then onwards it had 17, 23 and 21 minutes during successive intervals of approximately one hour, an average of $20 \cdot 3$ minutes.

The first subculture from it showed in its first three hourly intervals 20,22 and 19 minutes respectively, that is, an average generation time of 20.3 minutes, and it is to be observed in this case that no diminution of the generation time occurred during the second hour of the development of this subculture, but its rate was identical with the first hour of its growth within the error of the experiment.

From the parent culture a second subculture was made three hours five minutes after its inoculation when it was found that the parent and the subculture grew at similar rates for both the first and second following hours.

Since all the six experiments behaved in this way I suggest that in the case of both Rahn's and Coplans' work the restraint found was due to the fact that the parent culture had really passed its period of maximum growth.

Chart 3 shows, in the case of the experiments detailed, the logarithms of the parent culture and its two subcultures plotted against time. In some of these experiments it was found that the generation time of the subculture during the first quarter of an hour appeared slightly longer than the average minimum, but it was also found to be occasionally shorter, so that it seems inadvisable to lay too great stress on rates of growth calculated from these relatively short periods. If Coplans' 


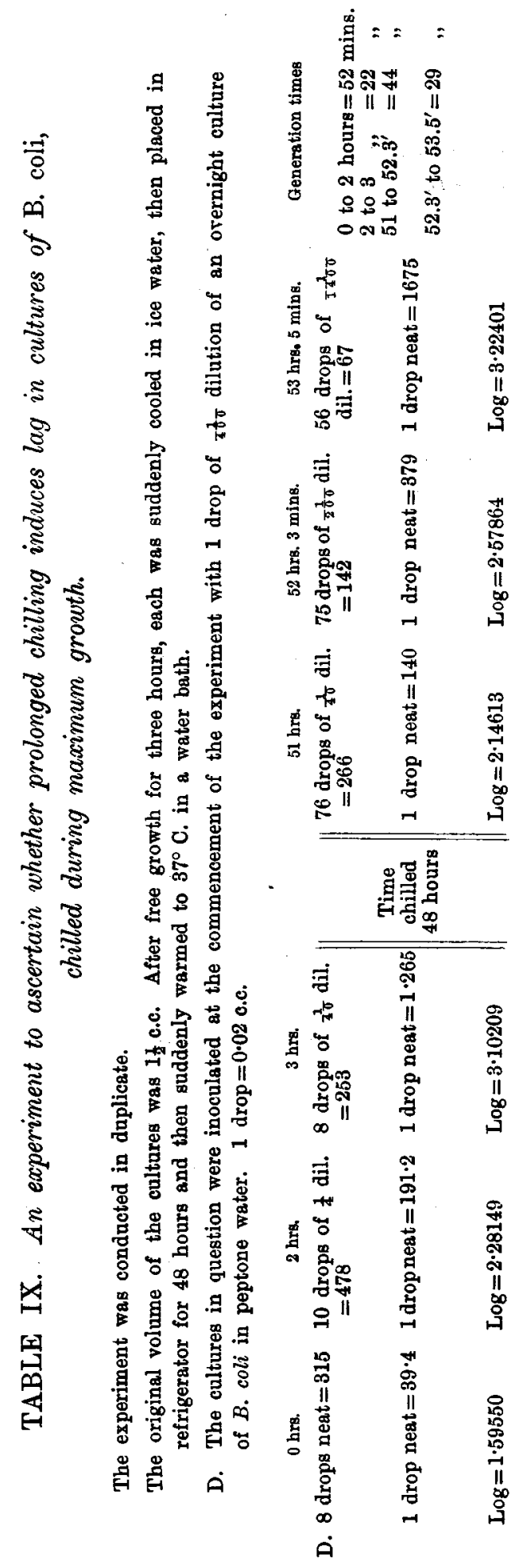


TABLE X. An experiment to ascertain if subculture during maximum growth is followed by a lag.

B. coli Escherich. Medium = peptone water.

\begin{tabular}{|c|c|c|c|c|c|c|}
\hline \multirow[b]{2}{*}{$\begin{array}{l}\text { Times, } \\
\text { mins. }\end{array}$} & \multicolumn{2}{|c|}{ Culture A } & \multicolumn{2}{|c|}{ Subculture B } & \multicolumn{2}{|c|}{ Subculture C } \\
\hline & $\begin{array}{l}\text { No. } \\
\text { per c.c. }\end{array}$ & $\begin{array}{c}\text { No. of } \\
\text { colonies } \\
\text { counted }\end{array}$ & $\begin{array}{l}\text { No. } \\
\text { per c.c. }\end{array}$ & $\begin{array}{c}\text { No. of } \\
\text { colonies } \\
\text { counted }\end{array}$ & $\begin{array}{l}\text { No. } \\
\text { per c.c. }\end{array}$ & $\begin{array}{c}\text { No. of } \\
\text { colonies } \\
\text { counted }\end{array}$ \\
\hline 0 & 328 & 105 & - & - & - & - \\
\hline 15 & 231 & 74 & - & - & - & - \\
\hline 30 & 209 & 67 & - & - & - & - \\
\hline 45 & 275 & 66 & - & - & - & - \\
\hline 60 & 420 & 101 & - & - & - & - \\
\hline 120 & 1900 & 76 & - & - & - & - \\
\hline 122 & - & - & 479 & 115 & - & - \\
\hline 135 & - & - & 835 & 167 & - & - \\
\hline 150 & - & - & 1355 & 190 & - & - \\
\hline 165 & - & - & 2010 & 201 & - & - \\
\hline 180 & - & - & 3450 & 207 & - & - \\
\hline 185 & - & - & - & - & 975 & 234 \\
\hline 192 & 32,300 & 323 & - & - & - & - \\
\hline 200 & - & - & - & - & 1788 & 322 \\
\hline 215 & - & - & - & - & 2191 & 263 \\
\hline 230 & - & - & - & - & 4425 & 354 \\
\hline 245 & - & - & - & - & 5830 & 350 \\
\hline 259 & 236,000 & 590 & - & - & - & - \\
\hline 262 & - & - & 46,300 & 463 & - & - \\
\hline 327 & - & - & $-^{\circ}$ & - & 97,800 & 978 \\
\hline 328 & - & - & 528,250 & 634 & - & - \\
\hline 329 & $2,255,000$ & 902 & $\cdots-$ & - & - & - \\
\hline $\begin{array}{c}\text { Time } \\
\text { interval, } \\
\text { mins. }\end{array}$ & $\begin{array}{c}\text { Generation } \\
\text { time, } \\
\text { mins. }\end{array}$ & $\begin{array}{c}\text { No. of } \\
\text { generations }\end{array}$ & $\begin{array}{c}\text { Generation } \\
\text { time, } \\
\text { mins. }\end{array}$ & $\begin{array}{c}\text { No. of } \\
\text { generations }\end{array}$ & $\begin{array}{c}\text { Generation } \\
\text { timee, } \\
\text { mins. }\end{array}$ & $\begin{array}{c}\text { No. of } \\
\text { generations }\end{array}$ \\
\hline 0 to 120 & 47 & $2 \cdot 53$ & - & - & - & - \\
\hline 60 to 120 & 27 & $2 \cdot 17$ & - & - & - & - \\
\hline 120 to 192 & 17 & $4 \cdot 08$ & - & - & - & - \\
\hline 122 to 180 & - & - & 20 & $2 \cdot 85$ & - & - \\
\hline 122 to 328 & - & - & $20 \cdot 3$ & $10 \cdot 10$ & - & - \\
\hline 180 to 262 & - & - & 22 & $3 \cdot 75$ & - & - \\
\hline 185 to 245 & - & 一 & - & - & 23 & $2 \cdot 58$ \\
\hline 192 to 259 & 23 & $2 \cdot 86$ & - & - & - & - \\
\hline 245 to 327 & - & - & - & - & 20 & 4.07 \\
\hline 259 to 329 & 21 & $3 \cdot 25$ & - & - & - & - . \\
\hline 262 to 328 & - & - & 19 & $3 \cdot 51$ & - & - \\
\hline
\end{tabular}


result had obtained and a slowing of growth equal to one minimum generation time had occurred, we would have found the generation time of the first hour in each experiment to be about 30 minutes, i.e. we would have had two generations during the first hour as against three in the second. All my experiments are incompatible with such a result.

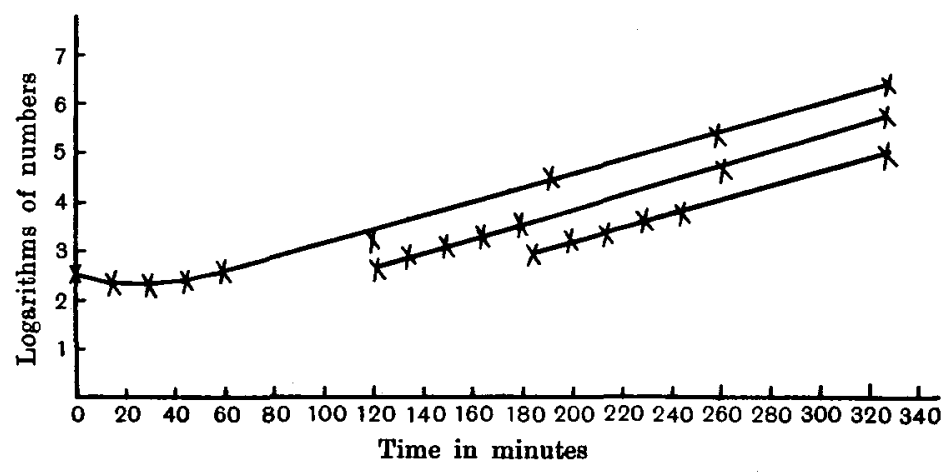

Chart 3 (with Table I). Subculture during maximum growth and lag.

\section{Effect of temperature on lag.}

Lane-Claypon (1909) states that the latent period with $B$. coli extended from one to six hours as the temperature fell from $42^{\circ}$ to $20^{\circ} \mathrm{C}$. During this fall of temperature the generation time lengthened four times. The published protocols of her experiments unfortunately do not deal with the lag period. It is obvious that lag and rate of growth are both affected by temperature but according to Lane-Claypon's unpublished figures not precisely equally. There do not appear to be many experimental data published on this subject. H. Chick (1913) however has shown that in serum the lag at temperature $40^{\circ} \mathrm{C}$. is about one hour while at $20^{\circ} \mathrm{C}$. it is $4 \frac{1}{2}$ hours. It is rather interesting to note that the generation time of $B$. coli in serum was affected very similarly.

Preliminary work suggests that the lag of $B$. coli, growing in peptone water at $20^{\circ} \mathrm{C}$., is about six hours when inoculated from a parent culture of 15 hours. But as the experiments are only preliminary I propose to delay publication until I have more exact data on the subject. 
Effect of age of parent culture on lag.

Max Müller (1895) demonstrated that an old parent culture was associated with a long lag in the subculture. This has been confirmed by Rahn (1906) and Coplans (1909).

I have repeated this work and have had no difficulty in observing a marked difference in the lags of subcultures made from cultures of $B$. coli grown at $37^{\circ} \mathrm{C}$. for 17 hours and four days respectively. The parent cultures and subcultures were grown in each case at $37^{\circ} \mathrm{C}$. It will be noticed on consulting the charts illustrative of this point that there appears to be no definite prolongation of the lag in passing from a parent culture of four days, to one of 12 days. Indeed, in the case recorded, it appears slightly less pronounced in the latter. In another experiment four day and eight day parent cultures gave the same lag on subculture. The fact that prolongation of lag is a marked feature in comparatively young cultures (of 17 hours to four days in the case of $B$. coli growing at $37^{\circ} \mathrm{C}$.), while as the culture gets older no further prolongation occurs, is of considerable importance from the theoretical standpoint. This fact lends no support to the view that lag is an expression of injury. Table XI shows the details of one out of two experiments performed to elucidate this point. An endeavour was made in each experiment to obtain frequent observations at the critical periods.

The nature of the medium and its effect upon lag.

In subculturing from one medium to another it is common knowledge that long periods of lag in growth may occur, apparently depending on some adaptation.

If adaptation has been secured, however, by subculturing two series of the same organism, each series on a special medium, it may be found that the lag obtained on subculture on the respective media varies with the medium. In illustration of this point I may mention that Coplans has shown that subculture of a dulcite peptone water culture of $B$. coli on to dulcite peptone water gives a longer lag than a subculture of a peptone water culture on to peptone water, though the parent cultures are of the same age. I have repeated this experiment and have been able to confirm the result. See Table XII and Chart 4 .

This effect of dulcite in my experiment is not due to the large size of the inoculation in the case of the dulcite culture, as an examination 


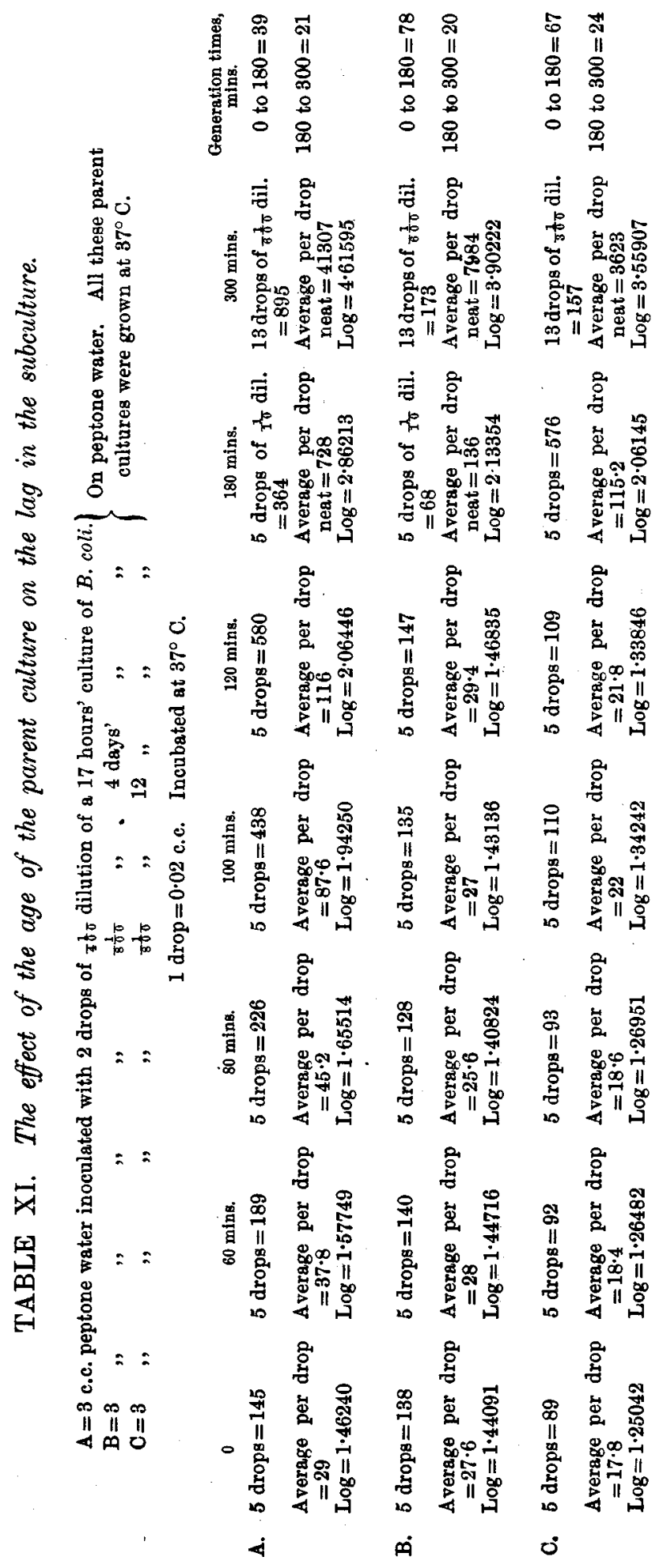




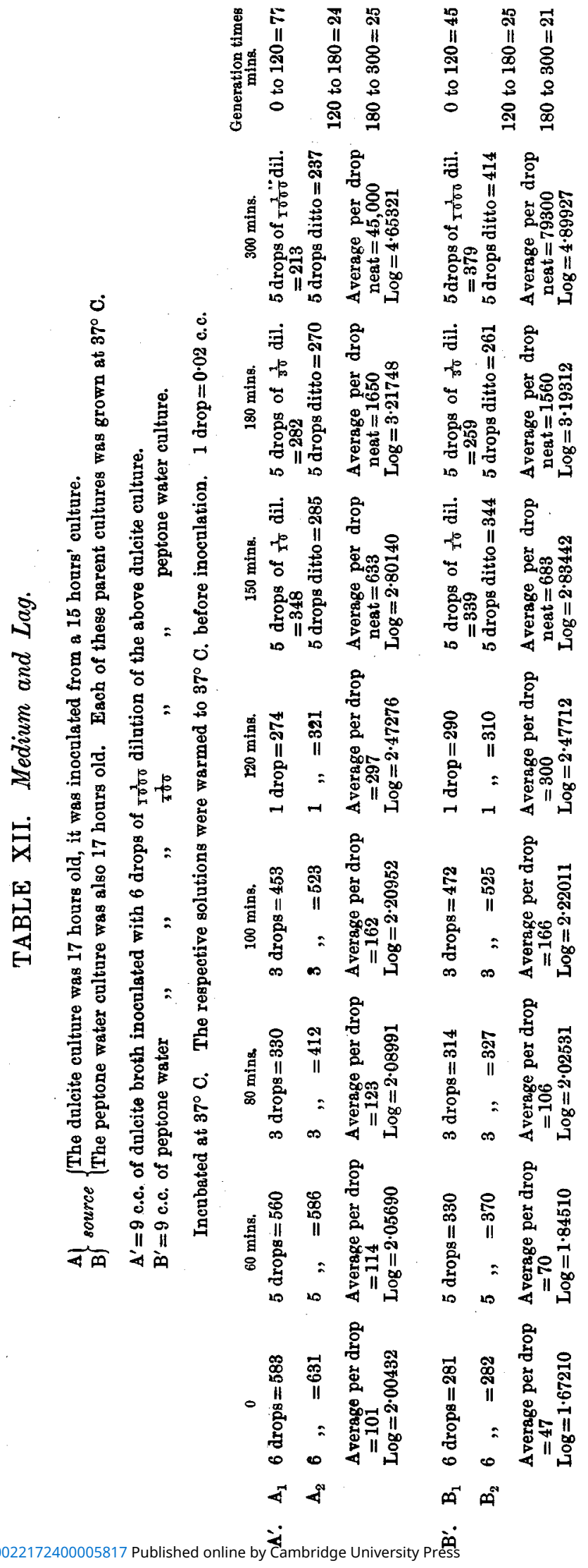


of the figures on that subject shows that we are dealing with inoculations of such a size as to be but slightly affected by this factor. It will be noted, however, that the dulcite parent culture had about 5.3 times the population of the peptone water parent culture and this may have rendered it similar to a peptone water culture of much greater age. Age of parent culture has already been shown to prolong lag.

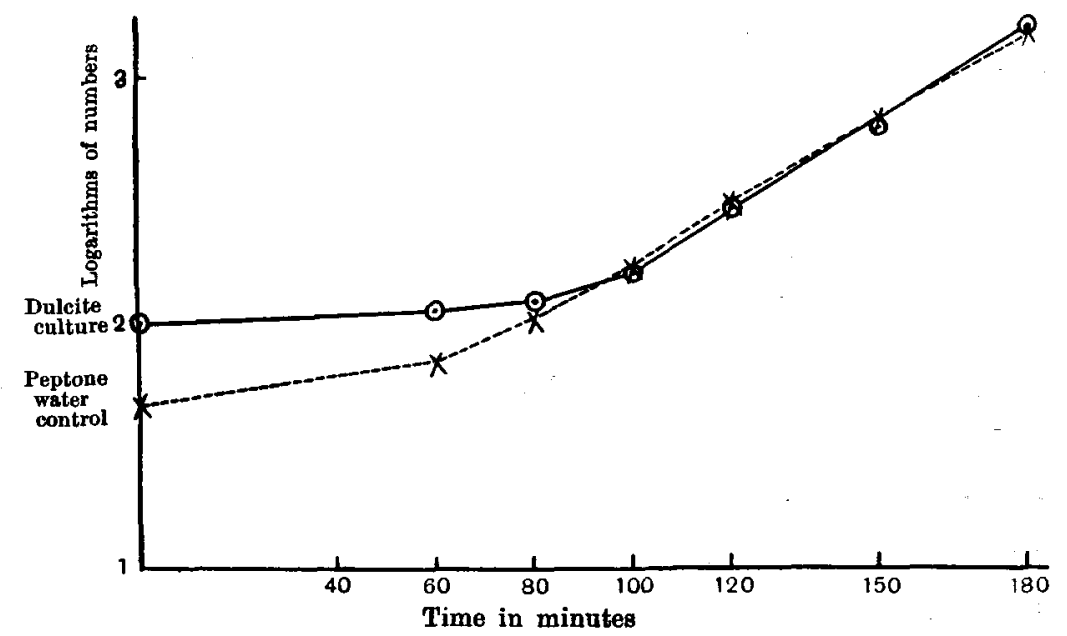

Chart 4. The nature of the medium and its effect upon lag.

Heat-stable bacterial products and lag.

It has been suggested that in the supernatant fluid of centrifuged cultures there are heat-stable bodies which may affect lag. Experiments performed to test this point gave no indication that any marked effect on lag resulted.

Table XIII and Chart 5 give the details of one such experiment from which it will be seen that these products have but little effect on lag, and apparently the slight effect they do exercise is in the direction of lengthening it.

The details of the experiment and technique employed appear in the table.

\section{Inhibiting agents.}

Eijkmann (1904) and others have long held the view that inhibition of growth in cultures is due to thermolabile inhibiting agents produced by the growth of the organisms, and it has naturally been thought that these bodies play an important part in the production of bacterial lag. 


\section{TABLE XIII. Heat-stable Products.}

$A=1 \frac{1}{2}$ c.c. of overnight culture of $B$. coli in peptone water centrifuged to Fach of $A, B, C$, and $D$ remove the organisms and heated $15 \mathrm{mins}$, at $100^{\circ} \mathrm{C}$.

$B=1 \frac{1}{2}$ c.c. of overnight culture of $B$. coli in peptone water heated as above but without previous centrifuging.

$C=1 \frac{1}{2}$ c.c. of overnight culture of $B$. coli in peptone water centrifuged after heating for 15 mins. at $100^{\circ} \mathrm{C}$.

$D=1 \frac{1}{2}$ c.c. of ordinary peptone water as control. was inoculated with 1 drop of $x_{\pi}$ dilution of the original overnight culture of $B$. coli from which they themselves had been derived.

1 drop $=0.02$ c.c.
A. 4 drops $=183$
120 mins.
240 mins.
360 mins.
Generation times, 6 drops of
$=1003$
Average per drop Average per drop
Average per drop
Average per drop
$=46$
$\log =1 \cdot 66276$ $=121$
neat $=1670$
neat $=22466$
$\log =4 \cdot 35141$
0 to $120=86$
$\log =3 \cdot 22272$
120 to $240=32$
$\log =\mathbf{2} \cdot 08279$
1 drop of $\frac{1}{10}$ dil. 1 drop of $1 \frac{1}{10}$ dil. $=218$ $=270$
B. 4 drops $=179$
Average per drop
4 drops $=\mathbf{5 3 8}$
$=45$
$\mathbf{L o g}=1 \cdot 65321$
Average per drop
Average per drop
Average per drop
neat $=2180$
neat $=27000$
Log $=2 \cdot 13033$
$\log =3 \cdot 33846$
$\log =4 \cdot 43136$
C. 4 drops $=152 \quad 4$ drops $=455$
1 drop of $\frac{1}{10}$ dil. 3 drops of $\frac{1}{160}$ dil. $=739$
Average per drop Average per drop

$$
=181
$$$$
=739
$$
$=38$
$\mathbf{L o g}=1.57978$

$$
\begin{aligned}
& =114 \\
& \log =2 \cdot 05690
\end{aligned}
$$
neat $=1810$
$\mathbf{L o g}=\mathbf{3} \cdot 25768$
Average per drop
neat $=24600$
$\log =4 \cdot 39094$
D. 4 drops $=150 \quad 4$ drops $=469$
Average per drop
neat $=\mathbf{3 8}$
Iog $=157978$
Average per drop
$=117$
9 drops of $\frac{1}{60} \mathrm{di}$ $=1222$
Average per drop
neat $=6800$
$\log =\mathbf{3} \cdot 83251$
1 drop
$=380$
Average per drop 120 to $240=20$ neat $=190000$ $\log =5 \cdot 27875$
0 to $120=76$
120 to $240=30$ 240 to $360=33$
0 to $120=76$
120 to $240=30$ 240 to $360=32$
0 to $120=74$ 240 to $360=25$

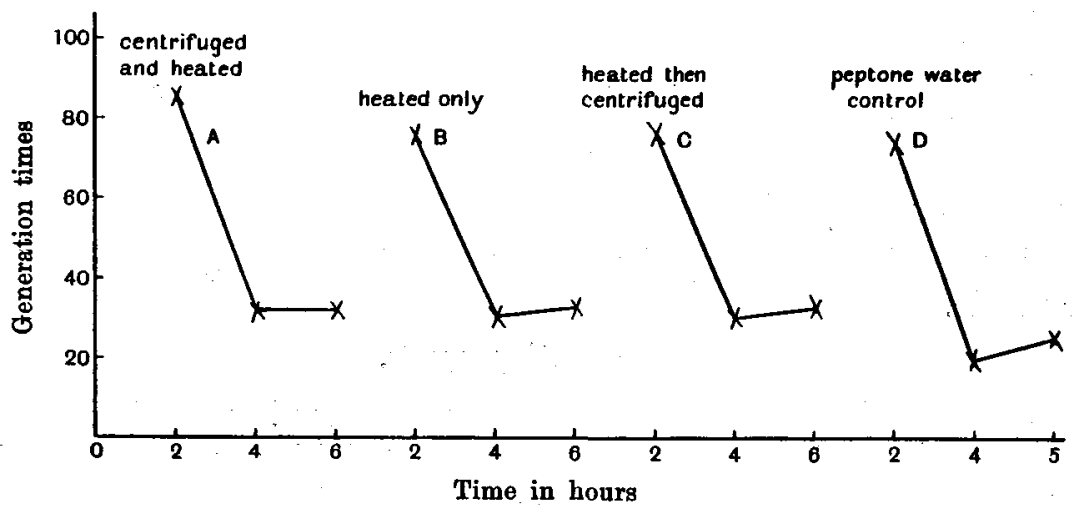

Chart 5. Heat-stable bacterial products and lag. 
The existence of these bodies and the part they play in bacterial inhibition have been the subject of warm controversy. Without coming to any decision with regard to these agents I made repeated attempts, by washing in saline and Ringer's fluid for one or two hours, to obtain seeding material from 17 hours' peptone water cultures which would be accompanied by no lag on subculture. I found that this method of treatment did not remove the lag. On the other hand after washing with saline the lag was slightly increased, while in the case of Ringer two hours immersion prevented subsequent growth. The proper salt ratio of a fluid with which to wash $B$. coli without injury to it, has probably not been found, so that in this experiment one may be simply substituting injury for the ordinary cause of lag. I do not propose to give the details of these experiments, the interest of which would have lain in the finding of a positive result.

\section{TABLE XIV. ExP. A. Supernatant and Lag.}

B. coli in autoclaved peptone water $\left(1 \%+\cdot 5 \%\right.$ salt), grown overnight (24 hours) at $37^{\circ} \mathrm{C}$. The organisms were centrifuged off and the supernatant plated and found to contain 148 organisms in 1 drop of $1_{1}^{1}$ dilution. This stood in cold room at $0^{\circ} \mathrm{C}$. overnight. It was plated out next day and gave the numbers given below at time 0 . It was then warmed and placed in the incubator at $37^{\circ} \mathrm{C}$. and counted as indicated. A culture of $B$. coli in peptone water has after 20 hours growth a generation time varying from approximately six hours upwards. 1 drop $=0.02$ c.c.

0

A. 1 drop of $\frac{1}{10}$ dil. $=156$

$1, ",=168$

$1, ", \quad, \quad=166$

Average per" drop neat

$=1630$

$\log =\mathbf{3} \cdot 21219$
120 mins.

\begin{tabular}{|c|c|c|}
\hline 1 drop o & 6 & $=89$ \\
\hline 1 ", & ," & $=105$ \\
\hline $\begin{array}{l}3 \text { drops } \\
3\end{array}$ & 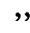 & $\begin{array}{l}=256 \\
=981\end{array}$ \\
\hline 3 A , & & $=281$ \\
\hline $\begin{array}{r}=3650 \\
\mathrm{Log}=3\end{array}$ & & \\
\hline
\end{tabular}

360 mins.

1 drop of $\frac{1}{5000}$ dil. $=19$
1 ,,$\quad 24$
5 drops ,", $=109$
5 , , $=138$
Average per drop neat
$=120830$
Log $=5.08207$

Generation times, mins.

0 to $120=103$

120 to $360=47$

\section{Exp. B. Supernatant and Lag.}

B. coli was grown on peptone water (1\% peptone $+5 \%$ salt) for 4 days at $37^{\circ} \mathrm{C}$, then centrifuged and placed in the cold room overnight. The rest of this experiment was conducted as $A$ and gave the following result.

B. 1 drop of $\frac{1}{10}$ dil. $=14$

5 drops,$"=72$

Average per drop neat $=143$

$\log =2 \cdot 15534$
120 mins.

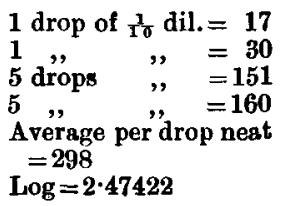

495 mins.

1 drop of $\frac{1}{1000}$ dil. $=85$

$1,, ",=70$

10 drops $\quad " \quad=666$

10 ," $",=806$

Average per drop neat

$=73950$

Log $=4 \cdot 86894$
Generation times,

0 to $120=113$

120 to $495=47$ 


\section{Growth on supernatant and lag.}

If a peptone water culture of $B$. coli be centrifuged it is found that the few bacteria remaining in the supernatant commence to grow again at a quick rate but not without a period of lag. I have not submitted full quantitative data dealing with this fact because those I have obtained so far are of a preliminary character. In Table XIV are given the data of two such experiments, each done in duplicate, from which it is seen that a marked lag occurred in each case. The lag was of so pronounced a character that it probably could not be accounted for by the fact that the culture had been overnight in the cold room. Further, after two hours, the growth of the residual organisms becomes so rapid as to suggest that no very powerful inhibiting agents of any kind are present.

\section{Summary of Results.}

(1) If $B$. coli be subcultured into another sample of the same medium when growing at full pace, it will continue to grow at the same pace.

(2) If the maximum rate of growth be interrupted by a short application of cold, growth will recommence without lag on the temperature being raised. If the cold be long continued, lag will tend to reappear.

(3) Differences in the size of inoculum have practically no effect on lag in the case of large inoculums, in the case of small ones, on the other hand, diminution of the seeding has the effect of lengthening lag, and this lengthening effect is more marked the smaller the seedings become.

(4) Lowering the temperature lengthens the lag. The effect is very similar to the effect on growth.

(5) The older a parent culture (within limits) the longer the lag.

(6) The length of lag varies with the medium even if adaptation has been arranged for beforehand.

(7) Heat-stable products in $B$. coli cultures on peptone water have, in the case of overnight cultures, but little effect on lag.

(8) After washing the bacteria for two hours with saline in order to remove possible inhibiting agents, it was found that the lag, on subculture, still occurred and was indeed slightly longer.

(9) If a peptone water culture of $B$. coli be centrifuged, it is found that the few bacteria remaining in the supernatant commence to grow again at a quick rate but not without a period of lag. 


\section{Discussion of modern views on the subjoct.}

It has been suggested that:

(a) Something must be secreted into the medium before maximum growth occurs. Against this is the fact that subculture when growth is maximal is followed by maximal growth. On the other hand this factor may come in in the case of subcultures of slowly growing parent cultures; it might account for the fact that increase of size of inoculum tends to diminish lag.

(b) Any change from one medium to another requires adaptation and this is attended with initial slow growth.

This is not the explanation of any of the lags with which we have been dealing as no such change occurred in any case.

(c) The osmotic pressure of the parent culture medium is different from that of the new sample of the same medium. A marked lag, as we have seen, is however present in the case of the residual organisms left after centrifuging a 24 hours' culture of $B$. coli. This may be partly accounted for by the exposure to cold of the culture after centrifuging, but a reference to previous experiments detailed in this paper shows that this is quite insufficient to account for the total lag, and equally it cannot be explained on variations of osmotic pressure of the medium.

(d) The presence in the medium of the end products of metabolism are essential to maximal growth. It has been shown that emulsin splits salicin better in the presence of saliginin and glucose. This cannot have a general application however in the case of bacterial lag for the same reason as negatives hypothesis $(a)$.

(e) The transferred organism may not be viable, some of them may die; since, however, all our initial populations are counted by their power to grow in agar plates, this factor probably does not come in.

$(f)$ The transferred organisms may agglutinate. This factor comes in to some extent in dealing with serum and milk as culture media, but $I$ was unable to obtain any evidence of it in the case of $B$. coli growing on peptone water.

(g) That the organisms are injured by the accumulated metabolic products of the parent culture. In the case of $B$. coli growing on peptone water, however, this does not appear to be the complete explanation, since the residual organisms after the culture is centrifuged are able to attain a generation time of $\mathbf{4 7}$ minutes though the whole culture had a generation time of about six hours. 
(h) That the inoculum consists of organisms having individually different powers of growth and that during the lag the selection of a quick growing strain occurs in response to some selecting agent in the peptone. This would present an analogy to the selection of bacteria which goes on in media containing a fermentable sugar.

Under this scheme a subculture made during the lag would show a lag somewhat shorter than the lag of the parent culture, while a subculture made during the so-called logarithmic period would show no lag. This as a matter of fact is what happens. At the end of the logarithmic period a selection would again take place in response to some other constituent of the culture and so conditions would result which would entail lag on subculture. Much may be said for this view, reasoning by analogy, but if it were true one would naturally suppose if two subcultures were made from the same parent culture marked differences in the rates of growth might occur between them, especially if the seedings were small. Now a reference to the experiments on size of inoculation and lag will show that the duplicate tubes always grew about the same rate and the differences between the members of pairs with small seedings were no greater than between the members of pairs with large seedings. I have obtained the same result in many unpublished experiments. This does not favour the existence of great variability in power of growth in the population.

(i) In addition to these various views it appeared desirable to exclude the possibility of inertia on the part of the bacteria, and for this purpose the chilling experiments were done. These experiments show that stoppage of growth by cold does not of necessity occasion a lag before subsequent maximal growth is attained.

(j) It has been shown that an induction period occurs in certain chemical reactions and in some instances at least this has been shown to be due to the fact that the reaction takes place in stages. It is quite obvious that if substance $C$ is produced not from substance $A$ directly, but from an intermediate substance $B$, then the maximum production of $C$ will occur, not at the commencement of the reaction when none of $B$ is present but only after $B$ has reached maximum concentration. It seems possible that in bacterial lag we have a phenomenon that may be explained on this purely chemical basis. I venture to suggest :

(1) Some of the constituents of the bacterial protoplasm are probably synthesized in steps, perhaps by a succession of enzymes.

(2) Maximum growth presupposes the optimum concentration 
attainable by the bacterium, of the intermediate bodies in the steps of the syntheses.

(3) When bacteria have stopped growing these intermediate bodies tend to diffuse out into the medium or disappear in some other way and their concentration in the bacteria falls.

(4) That transfer to a new medium is only followed by maximal growth when these intermediate products have again attained optimum concentration in the organism.

This view of lag would be in accord with the known facts. It might also account for that portion of the lag which can be removed by increasing the inoculum and which may depend on the absence of intermediate products in the medium. Since, however, no lag occurs if an organism be transferred while growing at maximum rate, we must look upon their presence in the medium as being of secondary importance. This absence of lag under these conditions would appear to be due to the fact that the intermediate bodies are present in the transferred organisms in optimum concentration. The effect of the cold may be explained on this hypothesis in this way-if the cold be evanescent these bodies have not time to disappear by diffusion or otherwise, if it be long continued they have, and on that account lag tends to reappear.

\section{REFERENCES.}

Buchner, Longard and Rieduin (1887). Ueber die Vermehrungsgeschwindigkeit der Bakterien. Centralbl. f. Bakteriol. II. 1.

Chick, H. (1912). The Bactericidal Properties of Blood Serum. Journ. of Bygiene, XII. 414.

Coplans, M. (1909, 1910). Influences affecting the growth of Micro-OrganismsLatency: Inhibition: Mass action. Journ. of Pathology, xIv.

EIJKMANN (1904). Ueber thermolabile Stoffwechselproducte als Ursache der natürlichen Wachstumhemmung der Microorganismen. Centralbl. f. Bakteriol. Abt. I, Originale, xxxviI. 436.

Lane-Claypon (1909). Multiplication of Bacteria and the Influence of Temperature and some other conditions thereon. Journ. of Bygiene, Ix.

Müller, Max (1895). Ueber den Einfluss von Fieber temperaturen auf die Wachstumgeschwindigkeit und die Virulenz des Typhus Bacillus. Zeitschr. f. Hygiene und Infektionskr. xx. 245.

RAHN (1906). Ueber den Einfluss der Stoffwechselprodukte auf das Wachstum der Bacterien. Centralbl. f. Bakteriol. Abt. II, Originale, xvi. 